\title{
Structuring an Early Clinical Experience for Pharmacy Students: Lessons Learned from the Hospital Perspective
}

\author{
Margaret L Ackman and Tania M Mysak
}

\section{INTRODUCTION}

\begin{abstract}
lberta Health Services-Edmonton Area is a division of a

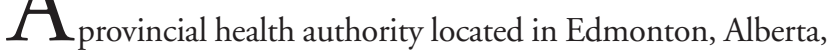
serving a population of about 1 million people. It consists of 13 facilities with over 2500 beds, in addition to outpatient clinics and public health offices. Regional Pharmacy Services (RPS), staffed by nearly 180 pharmacists and more than 190 technicians, provides clinical and distribution services. RPS provides a significant proportion of institutional rotation sites for students of the Faculty of Pharmacy and Pharmaceutical Sciences (FPPS) at the University of Alberta.
\end{abstract}

FPPS recently revised the undergraduate curriculum to a modular form and integrated Alberta College of Pharmacists internship hours with FPPS-required experiential training. Until this curricular revision, the first structured hospital experience for undergraduate students did not occur until the final professional year of the program, but a 2-week hospital rotation for second-year pharmacy students has now been added to the curriculum. The first of these rotations was offered in the spring of 2006.

The overall goal of this rotation is "to provide the student with an opportunity to participate in the role of the pharmacist in a collaborative and multidisciplinary institutional setting". The objectives and activities of the rotation include understanding patients' drug-related needs; understanding pharmacists' legal, ethical, and professional responsibilities; conveying information and recommendations related to drugs and drug use; and learning about drug distribution. ${ }^{1}$

The development of this rotation posed a number of challenges for RPS, particularly with respect to providing opportunities for students to participate in the pharmacist's role. Given the modular nature of the curriculum, the students' therapeutic knowledge base upon completion of their second year is limited to certain areas (Table 1). In addition, the limit-
Table 1. Therapeutic Modules Completed by Second-Year Undergraduate Students

Dermatology and eyes, ears, nose, and throat

Urology and nephrology

Hematology

Gastrointestinal system and nutrition

Pediatrics and geriatrics

Immunology

Cardiology

Pain

ed duration of the rotation (10 working days) and the requirement to cover some aspects of drug distribution (including medication safety) presented challenges for preceptors accustomed to the 6-week academic rotations of the final year. Finally, because 2006 was the first year for this new academic rotation, the RPS staff members did not have a full understanding of FPPS's expectations for experiential learning and contribution to patient care during their initial planning.

Our goal was to develop and evaluate a new hospital rotation designed to introduce the clinical role of the hospital pharmacist and provide direct patient care experience to secondyear pharmacy students. This report describes our experiences with this rotation and outlines the lessons that we learned.

\section{DEVELOPMENT AND DESCRIPTION OF THE STRUCTURED EXPERIENCE}

The RPS clinical practice leaders have overall responsibility for any students undertaking experiential rotations within RPS. It was this group of 9 individuals who met to plan the first set of second-year student rotations. We decided to model the rotation on the principles of our summer clinical program for pharmacy students, which has been described in detail elsewhere. ${ }^{2}$ In brief, the summer students participate in a series 
of defined clinical tasks for which they are explicitly trained in a workshop setting; the training is followed by observation of pharmacists conducting the task, and the students then conduct the defined clinical task while being observed by a pharmacist. Following evaluation of their skills, the students conduct the tasks under indirect supervision. This approach allows the summer students to participate in direct patient care and to make a contribution at the level of individual patients. In our experience, this model provides students with a more accurate and realistic impression of hospital pharmacy practice and the impact of pharmacists in the institutional setting than is possible with shadowing experiences. The length of the new 2-week rotation allows insufficient time for mastery of skills, to the point where the student could conduct tasks under indirect supervision. Nonetheless, we wanted to create a rotation in which the students would feel that they were making a difference to patients.

FPPS incorporated strategies from the summer student program into the second-year rotation, including an oncampus orientation before the start of the rotation. The FPPS orientation was originally scheduled for 18 contact hours, but was later decreased to 12 contact hours. ${ }^{2}$ This orientation consists of didactic and interactive seminars on a broad variety of topics, including the patient chart and the importance of documentation, medication reconciliation, medication safety, communication strategies, electronic patient information, approaches to patient care, and drug information. In addition, the FPPS course requirements for this rotation are reviewed, and professionalism and confidentiality are discussed.

Students were assigned to their rotation sites by means of an internal computer matching program within FPPS. The initial iteration of this 2-week rotation (Table 2), which took place in 2006, began with a 1-day "regional orientation". The orientation consisted of teaching sessions organized by RPS related to the specific clinical activities and skills that students were expected to perform during their rotation. These skills included conducting medication counselling and seamless care activities, taking medication histories, performing allergy assessments, and converting from intravenous to oral medications when appropriate; guidelines were also provided regarding documentation. The intent of this day-long session was to give the students a consistent process for conducting each defined clinical task. Training included an explanation of each clinical program overall and the student's expected role. The focus of the training was on practical application of theoretical knowledge to direct patient care and use of clinical tools or algorithms provided by either RPS or FPPS. This process included all aspects of the task and supporting work such as preparation for discharge counselling, creation of medication calendars, seamless care activities with community pharmacists, and documentation. The remainder of the orientation day was spent with a preceptor, observing and practising clinical skills at the preceptor's practice site. While there were no specific objectives regarding interprofessional exposure, such interactions were expected to occur as part of the preceptor's normal scope of activities. Two half-days were scheduled to address FPPS requirements for information and recommendations related to drugs and drug use: one focused on searching the literature and the other on using the electronic resources available within RPS, including parenteral compatibility information. The students were then given a number of drug information questions to be answered during

Table 2. Regional Rotation Outline

\begin{tabular}{lll} 
Day of 2-Week Rotation & \multicolumn{1}{c}{ 2006 Rotation } & \multicolumn{1}{c}{ Planned 2009 Rotation } \\
\hline Day 1 & Regional orientation & Regional orientation* \\
\hline Day 2 & $\begin{array}{l}\text { Site orientation } \\
\text { Job shadowing }\end{array}$ & Clinical activities \\
\hline Day 3 & $\begin{array}{l}\text { Regional drug information } \\
\text { Clinical activities }\end{array}$ & Clinical activities \\
\hline Day 4 & $\begin{array}{l}\text { Regional drug information } \\
\text { Clinical activities }\end{array}$ & Clinical activities \\
\hline Day 5 & Clinical activities & Clinical activities \\
\hline Day 6 & Regional distribution & Clinical activities \\
\hline Day 7 & Site distribution & \\
\hline Day 8 & Clinical activities & Clinical activities \\
\hline Day 9 & Clinical activities & Specialty practice visit† \\
\hline Day 10 & Specialty practice visitł & Clinical activities \\
\hline & Clinical activities & Clinical activities \\
\hline Day 1 now & Clinical activities & Clinical activities \\
\hline
\end{tabular}

*Day 1 now includes regional distribution and regional drug information sessions.

†This can be scheduled for any single morning or afternoon on day 7, 8, or 9 . Rural sites would have

2 specialty practice visits scheduled on a single day.

¥This could be scheduled for any single morning or afternoon on day 7 , 8, or 9 . 
the course of the rotation. Faculty requirements concerning drug distribution and medication safety were addressed through a half-day discussion, presented by RPS, of the various types of drug distribution systems in use throughout the region and the patient safety advantages and challenges associated with each. The students then returned to their assigned sites for the afternoon to see the distribution system in use at that site. For many students, this rotation would be their first exposure to institutional practice, so we included one half-day shadowing a pharmacist in the practice area to which they were assigned and one half-day shadowing a pharmacist in specialty practice, such as critical care or an ambulatory clinic.

The assessment of students was directed by FPPS and consisted of written evaluation forms completed by both students and preceptors, as well as ongoing verbal feedback. In addition, each student had to prepare a written assignment for submission to FPPS.

\section{IMPLEMENTATION, EVALUATION, AND LESSONS LEARNED}

The first time this rotation was offered, in 2006, a group of 36 students came to RPS following completion of their second year. The rotations took place in 2 consecutive 2-week blocks at a variety of sites within RPS. Thirty-seven students completed the rotation in 2007 and 44 in 2008.

To assess the quality of the rotation and to determine what changes were needed for future years, evaluative data were collected in each of the 3 years by RPS from students on the final afternoon of the rotation and from preceptors after all of the students had completed their rotations. These data were gathered in the form of verbal feedback during structured group discussions led by the clinical practice leaders. These discussions were separate from the evaluations that students and preceptors completed for FPPS as a requirement of the academic rotation. The students and pharmacists were aware that the feedback from the structured group discussions was for internal use by RPS for program development. All of the students attended these regional debriefing sessions. Attendance was not taken at the preceptor feedback sessions. However, attempts were made to follow up with individual pharmacists who were unable to attend.

The feedback from each year was collated annually and then independently reviewed and categorized into themes by the 2 authors. Any inconsistencies between the themes were resolved through discussion. Plans for the structured experience for the next year were modified on the basis of the current year's feedback. Highlights of the identified themes are outlined below, along with the alterations that were made to the program based upon this information.

\section{Knowledge Base}

The limited number of therapeutic areas to which students had been exposed at the time of their rotations (Table 1) posed a challenge for both students and preceptors. The students felt that it would have been helpful to limit the rotation to only those therapeutic areas that had been covered in the curriculum and felt challenged by their lack of therapeutic knowledge. At the same time, the preceptors would have valued receiving additional information from FPPS regarding reasonable expectations for therapeutic knowledge. They felt pressured because they did not have enough time available to "teach therapeutics".

These concerns reinforced the importance of focusing the rotation on the building of basic clinical skills rather than the application of therapeutic knowledge, as well as the importance of clearly outlining this focus to both students and preceptors. We now provide more explicit information to the preceptors, through a structured orientation session that takes place before each year's rotation, regarding the overall goal of the rotation (i.e., the building of clinical skills) and the level of therapeutic knowledge of the students. The concerns expressed about students' knowledge base have diminished significantly over the years.

\section{Patient Contact}

The selection of inpatient units for the rotation was based on where the students would have the greatest opportunity to interact with patients and to practise the skills set out as rotation objectives. The students reported that they would have liked to spend more time speaking with patients and that this had been the most rewarding aspect of the rotation. They felt that it was important to gain experience with different types of patients and various communication challenges. The preceptors felt that students performed well within their defined clinical tasks, such as performing allergy assessments and obtaining medication histories.

Interestingly, after the 2006 rotation, the preceptors reported that the students' expectations regarding chart documentation exceeded the preceptors' practice in this area. At the time, many preceptors did minimal chart documentation, opting instead to maintain pharmacy records for each patient. In addition, there was individual variation among preceptors in their assessment of situations requiring chart documentation. Although the patient contact aspect of the rotation has not changed, RPS has now developed a regional standard for documentation and a supporting series of educational modules, which were introduced in 2007. No comments about documentation were raised in 2008, which suggests that these standards closed the gap between students' expectations and practice. 


\section{Rounds}

According to the original plan for the rotations, each student was to attend daily bedside team rounds with the pharmacist, to observe the process of care as delivered by a multidisciplinary team. Unfortunately, such rounds do not take place in all practice areas to which students were assigned. The students felt that they should all have had the opportunity to attend daily bedside rounds. However, some students felt that rounds were not as useful as interactions with individual patients. On the basis of this feedback, it was decided that participation in daily bedside team rounds could be fulfilled as a specialty practice experience for students assigned to areas that did not have daily team rounds.

\section{Specialty Practice Visit}

The students' response to the specialty practice visits was overwhelmingly positive. In fact, the students desired more opportunities to see a variety of practices. They also described the specialty practices as "more interesting" than general practice. However, careful examination of their comments revealed that most students were focused on the medical specialty itself, rather than the role of the pharmacist within that practice setting.

Given the goals of the rotation and the importance of the student as a provider of direct patient care, rather than as an observer, specialty visits have been maintained as a half-day activity. Exceptions have been made, however, for students at rural sites, who are scheduled for 2 half-days (on the same day and at the same site, for logistical reasons) to give them exposure to both rounding and non-rounding practices. To increase exposure to different practice types and to ensure that all students participate in 2 half-day visits, in future years each student at an urban site will join another student at the same site but in a different practice setting for one half-day.

\section{Alignment with Faculty Activities}

As described above, FPPS provided an on-campus orientation before the start of the rotations. The content of this orientation was not available to RPS during planning for the initial rotation, and the preceptors felt that this would have been helpful. In addition, the students indicated that there was some overlap between the FPPS sessions and those provided by RPS. However, they also appreciated that some of the information provided by RPS, particularly related to medication reconciliation and allergy assessment, was not provided in the FPPS orientation. The students felt that the regional orientation provided by RPS was an excellent introduction to hospital practice and complemented the activities they undertook during their rotations.
The FPPS orientation is provided to all students, regardless of the site of their institutional rotations. Originally, Edmonton and area was the only health region in Alberta to provide an additional regional orientation and a structured regional rotation curriculum. Subsequent to our experience, other health regions have adopted portions of our program for their second-year rotations. As well, FPPS has abbreviated and reworked its orientation, a portion of which is now provided by RPS employees. The RPS orientation has been adapted accordingly, which has essentially allowed us to eliminate duplication between the RPS and FPPS orientations. This has allowed us to further decrease our orientation time and increase patient contact time. This collaboration with FPPS has greatly improved the quality of the students' experience. Even so, because RPS has specific objectives related to patient contact, we have elected to keep certain components (e.g., allergy assessments, medication counselling) as part of the RPS orientation.

\section{Regional Drug Distribution}

For the 2006 rotation, FPPS identified specific objectives and activities related to drug distribution, mainly focused on process. These were covered in a half-day regional didactic session focused on medication safety and a half-day of practical site-based activities. The students found the regional session uninteresting, and the preceptors felt that the students did not seem to understand the processes required for safe and effective drug distribution systems.

Considering this feedback and the fact that FPPS covers drug distribution theory in its curriculum, we initially kept the distribution session at a regional level, reworking it to be more practical (including a tour of a site). However, the students still felt that the information discussed during this session did not add to their knowledge base (as developed during the academic year). Therefore, in future years the distribution session will be shortened substantially, will focus on patient safety, and will be held during the orientation day.

\section{Drug Information}

An orientation to drug information was covered in 2 half-day sessions, one focused on searching the literature and the other on the electronic resources available within RPS. The students indicated that the session on electronic resources was very useful, but noted that literature searching had been adequately covered during the academic year. The preceptors' impressions were consistent with this feedback. They also indicated that the students needed more experience with commonly used tertiary references. On the basis of this information, the drug information session was initially shortened to a single half-day, focused entirely on available electronic resources. On the basis of further feedback, the session has been 
shortened again and will be offered during the regional orientation day.

\section{Preceptor Availability and Support}

After the 2006 rotations, the students reported that they liked their preceptors and felt that it would be ideal to have only one preceptor. However, some students felt that they had not received the required support from their preceptors. For their part, the preceptors reported that they had difficulty offering only direct patient care activities during the students' rotations. Some preceptors reported that their sites were underresourced for student rotations, which in some cases was reflected by inability to assign one preceptor for the duration of the student's rotation (for example, because of staff schedules and limitations, a student might have had to adjust to multiple preceptors over the short rotation).

For subsequent rotations, it was deemed important to be very explicit with preceptors concerning the level of support required by and expected for second-year students. We stressed that the focus of the rotation was on building clinical skills rather than teaching therapeutic content. We also worked more closely with the individual rotation sites to endeavour to have only one preceptor per student and to have that individual focused on direct patient care during the student's rotation. These objectives have generally been achieved in later years of the program.

Finally, given the structured orientation now provided to preceptors, a newly developed preceptor training module that was implemented in 2008, and increased familiarity with the rotation (in its third year, as of the time of writing), the preceptors now feel adequately prepared for these students, and their comments have been generally very positive.

\section{Logistics}

RPS services a large geographic area, and both students and preceptors indicated that travel between sites posed certain challenges, particularly for students based at the rural and suburban sites.

To minimize time lost due to travel and related requirements, sessions have been rescheduled, wherever possible, to avoid isolated half-day sessions. In addition, for students at the rural sites, 2 specialty practice visits were scheduled on the same day at one of the larger academic sites. This allowed us to address the issue of lack of daily bedside rounds at some sites (as described above) and also to include a specialty practice component. Furthermore, the planned consolidation of all regional sessions into one regional orientation day will further improve logistics. Regional debriefing sessions may also be eliminated in favour of site-based sessions.

\section{Rotation Length}

Students generally felt that the rotation was either of adequate length or was too short. However, those who found it too short acknowledged that it was the experience they enjoyed and wanted to continue and that no additional time was required to fulfill the course objectives. The students also commented on the logistical difficulties of scheduling a potentially longer rotation at the end of the academic year, which might overlap with summer employment opportunities. Rotation length is ultimately the domain of FPPS, and we have simply provided this feedback to them for their consideration.

\section{DISCUSSION}

Overall, the preceptors were pleased with the concept of earlier exposure of students to hospital practice and were supportive of this experience. The students learned what a hospital pharmacist does, how the team approach works in health care, the application of theory to practice, and the importance of life-long learning. The students also commented on the difference in a pharmacist's impact on patient care between the hospital setting and community practice. For RPS as an organization, the development of this new rotation has emphasized the importance of proactive communication and the setting of clear expectations for both students and preceptors.

The benefits and challenges of experiential education are considerable. As the need for experiential education increases, different strategies will be needed on the part of both academia and the health care system. It has been suggested that the role of pharmacy students on rotation and the quality of their contributions to patient care be reconsidered and more thoughtfully directed. ${ }^{3}$ This would require earlier exposure to patient care and a more direct role for students. A 4-week community-based pilot study for students in their first professional year evaluated this possibility of early direct patient care by students. ${ }^{4}$ Students and preceptors were consistent in identifying the most valuable activities as those involving direct student-patient interactions. The pilot study also demonstrated that even students early in their professional training could interact successfully with patients. ${ }^{4}$ Our experience was consistent with this conclusion, although a longer duration of rotation would be required to allow students to interact with patients under indirect supervision.

The University of Toronto has offered an early exposure to hospital practice for students in their second professional year. ${ }^{5}$ The objectives of that program were similar to those of the second-year rotation described here. However, the program was abbreviated, consisting of 2 half-day site visits; during each visit, the student observed one professional interaction and one patient interview. Assessments from both preceptors and 
students indicated that the program was too short and that more time should be allocated for the students to provide patient care. ${ }^{5}$ The length of our program addresses these concerns to some degree, and our program could serve as a benchmark for other programs contemplating early exposure within their curricula.

Another option for early exposure is a longitudinal rotation. Brown and others ${ }^{6}$ described a semester-long training program during the second professional year, consisting of one afternoon ( $4 \mathrm{~h}$ ) each week for 14 weeks, with the specific training activities for a given day determined by the site preceptor. Both students and preceptors reported significant benefit from the weekly site visits. ${ }^{6}$

Regardless of the length and logistics of an early exposure rotation, the consistent message remains that not only are students at this stage of their professional training capable of providing direct patient care, but also that provision of such training early in the curriculum is perceived as highly valuable by students and preceptors alike.

\section{Conclusions}

A 2-week rotation designed to introduce the clinical role of the hospital pharmacist and provide direct patient care experience to second-year pharmacy students is feasible and can be a positive experience for both preceptors and students. Lessons learned from the early years of this rotation were applied to refine and improve the experience for both preceptors and students over time. The lessons learned from our program may also be applied by other Alberta institutions that act as second-year experiential training sites, to standardize the experience for students.

\section{References}

1. Pharmacy 315 experiential learning course manual. Edmonton (AB): University of Alberta, Faculty of Pharmacy and Pharmaceutical Sciences; 2008 Apr/May/Jun.

2. Ackman ML, Schindel TJ. Lessons learned: a pilot program to cultivate a clinical role for pharmacy students. Can J Hosp Pharm 2007;60(5): 324-329.
3. Chase P. Rethinking experiential education (or does anyone want a pharmacy student?). Am J Pharm Educ 2007;71(2):article 27.

4. Wright DE, Cox CE, Koshy BS, Rae N. Direct-patient care activities of first-year pharmacy students in an early experiential program [abstract]. Am J Pharm Educ 2001;65(4 Suppl):102.

5. Battistella M, Seki J, Wong G, Arora V, Musing E. Development and evaluation of a student early hospital exposure program in a Canadian Bachelor of Science Pharmacy program. Am J Pharm Educ 2004;68(4):article 102.

6. Brown DL, Smith LS, Ferrill MJ. Preceptor acceptance of a longitudinal model for providing early experiential training in hospital pharmacy during a didactic semester [abstract 261D]. ASHP Midyear Clinical Meeting; 2005 Dec 4-8; Las Vegas (NV).

Margaret L Ackman, BSC(Pharm), PharmD, FCSHP, is a Clinical Practice Leader with Pharmacy Services, Alberta Health Services. She is also a Clinical Adjunct Professor, Faculty of Pharmacy and Pharmaceutical Sciences, University of Alberta, Edmonton, Alberta.

Tania M Mysak, BSP, PharmD, is a Clinical Practice Manager with Pharmacy Services, Alberta Health Services, and Clinical Adjunct Professor, Faculty of Pharmacy and Pharmaceutical Sciences, University of Alberta, Edmonton, Alberta.

\section{Address correspondence to:}

Dr Margaret L Ackman

Regional Pharmacy Services

Alberta Health Services

0G1.01 Walter Mackenzie Centre

8440-112 Street

Edmonton AB T6G 2B7

e-mail: margaret.ackman@albertahealthservices.ca

\section{Acknowledgements}

We would like to acknowledge the support of the pharmacists of Alberta Health Services-Edmonton Area and the students of the Faculty of Pharmacy and Pharmaceutical Sciences, University of Alberta. In addition, we would like to acknowledge Theresa J Schindel, Director of Outreach Education, Faculty of Pharmacy and Pharmaceutical Sciences, for her thoughtful comments and questions during the preparation of this manuscript. 